\title{
Piezoelectric and electroacoustic properties of Ti-doped AlN thin films as a function of Ti content
}

\author{
Enrique Iborra, José Capilla, Jimena Olivares, \\ Marta Clement \\ GMME-CEMDATIC-ETSIT \\ Universidad Politécnica de Madrid \\ Madrid, SPAIN \\ eiborra@etsit.upm.es
}

\author{
Valeriy Felmetsger \\ OEM Group Inc. \\ Gilbert Arizona, USA
}

\begin{abstract}
In this work we present the assessment of the structural and piezoelectric properties of $\mathbf{A l}_{(0.5-\mathrm{x})} \mathbf{T i}_{\mathrm{x}} \mathbf{N}_{0.5}$ compounds (titanium content $<6 \%$ atomic), which are expected to possess improved properties than conventional AIN films, such as larger piezoelectric activity, thermal stability of frequency and temperature resistance. Al:Ti: $\mathrm{N}$ films were deposited from a twin concentric target of $\mathrm{Al}$ and $\mathrm{Ti}$ by reactive $\mathrm{AC}$ sputtering, which provided films with a radial gradient of the Ti concentration. The properties of the films were investigated as a function of their composition, which was measured by electron dispersive energy dispersive X-ray spectroscopy and Rutherford backscattering spectrometry. The microstructure and morphology of the films were assessed by $X$-ray diffraction and infrared reflectance. Their electroacoustic properties and dielectric constant were derived from the frequency response of $\mathrm{BAW}$ test resonators. Al:Ti:N films properties appear to be strongly dependent on the Ti content, which modifies the AIN wurtzite crystal structure leading to greater dielectric constant, lower sound velocities, lower electromechanical factor and moderately improved temperature coefficient of the resonant frequency.
\end{abstract}

Keywords-AlN doped, AlTiN, BAW, Sputtering, Piezoelctric, Electroacoustic devices

\section{INTRODUCTION}

Aluminum nitride (AIN) is the preferred piezoelectric material for a broad range of thin films devices and applications requiring piezoelectric actuation, such as resonators for RF filters for telecommunications, sensors, or micro-electromechanical systems (MEMS). Due to its good piezoelectric properties and chemical stability, AlN is especially appropriate for operation in moderately harsh environments, such as relatively high temperatures or corrosive media. However, the increasingly demanding applications require the development of new piezoelectric materials with improved properties. It has been reported that the addition of some chemical elements to the AlN wurtzite structure enables modification and improvement of the material physical properties, since the deformation undergone by the lattice leads to an improved performance in a wide range of applications, including hard coatings and electroacoustic devices. Among the various elements investigated as possible candidates for modifying AIN properties we can cite boron, chromium, manganese, cupper and scandium. Not all of them provide improved properties; for example, AlN and boron alloys exhibit smaller electromechanical coupling factors in electroacoustic devices due to a decrease of the piezoelectric coefficients $[1,2]$. However, it has been verified that the addition of scandium produces a significant increase of the overall piezoelectric activity of the Al:Sc:N alloy [3], although other parameters, such the acoustic losses or the sound velocity, worsen [4]. Manganese and copper turn AlN into a magnetic material, but we have not found reported data regarding its piezoelectric behavior $[5,6]$. Chromium and AlN alloys have been extensively studied because $\mathrm{Al}: \mathrm{Cr}: \mathrm{N}$ films exhibit interesting magnetic properties [7]. Regarding the piezoelectric properties, the introduction of chromium atoms in the wurtzite AlN lattice to an atomic concentration of around $6 \%$ produces an increase of the lattice parameter accompanied by a rise of the $d_{33}$ piezoelectric coefficient; in contrast, the stiffness and, therefore, the sound velocity, decreases [8,9].

Al:Ti:N alloys with cubic crystalline structure possess a high resistance to friction and are commonly used as hard coating for cutting tools [10]. However, we have not found any reference dealing with either the mechanical properties of the wurtzite hexagonal phase, or the electric and piezoelectric properties of Al:Ti:N thin films. Ti is a transition metal that can fit the oxidation level to substitute $\mathrm{Al}$ in the wurtzite AlN structure; its lower electronegativity, compared to $\mathrm{Al}$, allows predicting a ternary compound with modified properties respect to pure AlN [11].

Sputtering is the most appropriate technique for growing AlN-metal ternary compounds because it provides good process control, high homogeneity along large area substrates and ease industrialization. However, sputtering is a very complex technique in which many process parameters, usually non-independent, critically influence the morphology and properties of the deposited films. The above mentioned ternary compounds can be achieved by different ways: the simplest one is the sputtering of a compound metallic target under a reactive atmosphere. However, this process provides a poor control over the final composition of the layer, since the ratio between the two metallic components can hardly be varied. A more versatile solution is the co-sputtering of two elemental targets under reactive atmospheres; in this case, a good control of the composition homogeneity along the substrate is essential. 
In this communication we investigate the effects of the addition of different amounts of titanium in the physical properties of AlN films. We carry out an extensive assessment of the properties of piezoelectric wurtzite $\mathrm{Al}: \mathrm{Ti}: \mathrm{N}$ compounds deposited by co-sputtering a double coaxial target of $\mathrm{Al}$ and $\mathrm{Ti}$ in alternate current configuration.

\section{EXPERIMENTAL TECHNIQUES}

\section{A. Film deposition}

Al:Ti:N piezoelectric films with thickness of $1 \mu \mathrm{m}$ were deposited on $150 \mathrm{~mm}-\mathrm{Si}$ (100) wafers. The wafers intended for piezoelectric characterization had been previously covered with an acoustic reflector consisting of a film of aluminum, as low acoustic impedance layer, and a film of molybdenum, as high acoustic impedance layer, which provided a suitable substrate for building bulk acoustic wave (BAW) resonators. A $100 \mathrm{~nm}-$ thick high quality (110)-oriented Mo film acted as the bottom electrode. All the films were deposited in an Endeavor-AT cluster tool from OEM Group equipped with dual-target S-gun magnetrons [12].

Briefly, the S-gun consists of two ring-shaped targets (diameters of 178 and $280 \mathrm{~mm}$ ) mounted concentrically on the same vertical axis. The inner target is made of $\mathrm{Al}$ and the outer of Ti. Each target has its own magnetic array with opposite polarities. An alternating current power of $40 \mathrm{kHz}$ applied between the two targets creates a plasma discharge at the conical face of each target. The power fed to each target can be adjusted separately by a power splitter module. Due to this dual-target arrangement, where each target alternates as a cathode and an anode, the ac powered S-gun is free from parasitic arcing and disappearing anode effects. This target configuration allows obtaining films with non-uniform composition in the radial direction, which are useful to study the variations of the properties as a function of the Ti content. Prior to $\mathrm{Al}_{(0.5-\mathrm{x})} \mathrm{Ti}_{\mathrm{x}} \mathrm{N}_{0.5}$ deposition, the substrate surface was treated with low energy Ar ions either in a separate planarized RF (13.56 MHz) etch module, producing a capacitively coupled plasma, or in the deposition module (in-situ etch). Deposition processes were performed either without external heating $\left(\mathrm{T}<300^{\circ} \mathrm{C}\right)$ or at elevated temperatures (up to $400^{\circ} \mathrm{C}$ ) using an external IR heater to warm the wafer before or during deposition. A complete description of the deposition process can be found in [13].

\section{B. Film characterization}

The composition of the Al:Ti:N films was assessed by combining Rutherford backscattering spectrometry (RBS) measurements and energy dispersive X-ray spectroscopy (EDX). Fig. 1 shows the RBS spectrum of a representative Al:Ti:N/Mo/ $/ \mathrm{SiO}_{2} / \mathrm{Si}$ stack. We assumed that the $\mathrm{N}$ content in the films was $\sim 50 \%$ in all cases, which was confirmed by RBS measurements. The composition of the films was therefore $\mathrm{Al}_{(0.5-\mathrm{x})} \mathrm{Ti}_{\mathrm{x}} \mathrm{N}_{0.5}$. The fitting to the experimental data in fig. 1 yielded a Ti content of $4.1 \%$ atomic $(x=0.041)$.

Infrared reflection was measured with a Fourier transform infrared (FTIR) Nicolet 5-PC spectrophotometer using nonpolarized light with an incident angle of $30^{\circ}$ in the $400 \mathrm{~cm}^{-1}$ -
$4000 \mathrm{~cm}^{-1}$ range with a spectral resolution of $2 \mathrm{~cm}^{-1}$. These spectra provide information about the chemical bonding configuration of the films and allow deducing their composition after calibrating the position of the $\mathrm{Al}(\mathrm{LO})$ band around $880 \mathrm{~cm}^{-1}$ with the RBS and EDX data.

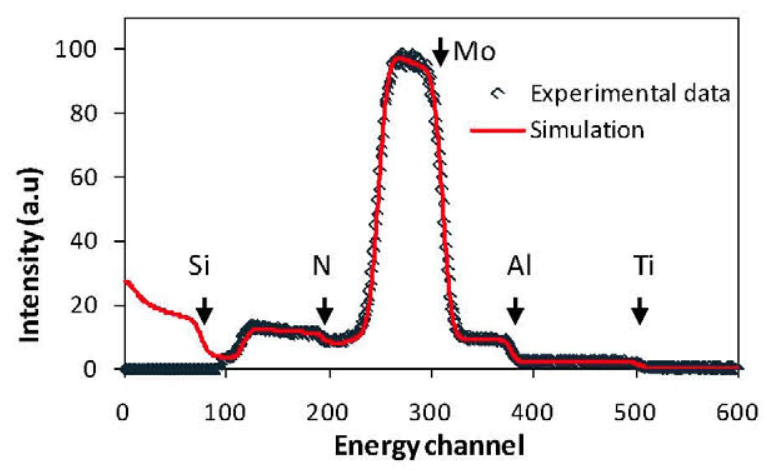

Figure 1. RBS spectrum of an $\mathrm{Al}_{(0.5-\mathrm{x})} \mathrm{Ti}_{\mathrm{x}} \mathrm{N}_{0.5}$ film with $\mathrm{x}=0.041$.

The absorption bands were fitted with lorentzian curves to accurately determine their position. Figure 2 shows the spectra of three representative $\mathrm{Al}_{(0.5-\mathrm{x})} \mathrm{Ti}_{\mathrm{x}} \mathrm{N}_{0.5}$ films with different $\mathrm{Ti}$ contents. It is worth noting that $\mathrm{Ti}-\mathrm{N}$ band is actually composed of two peaks in the films with the highest $\mathrm{Ti}$ contents.

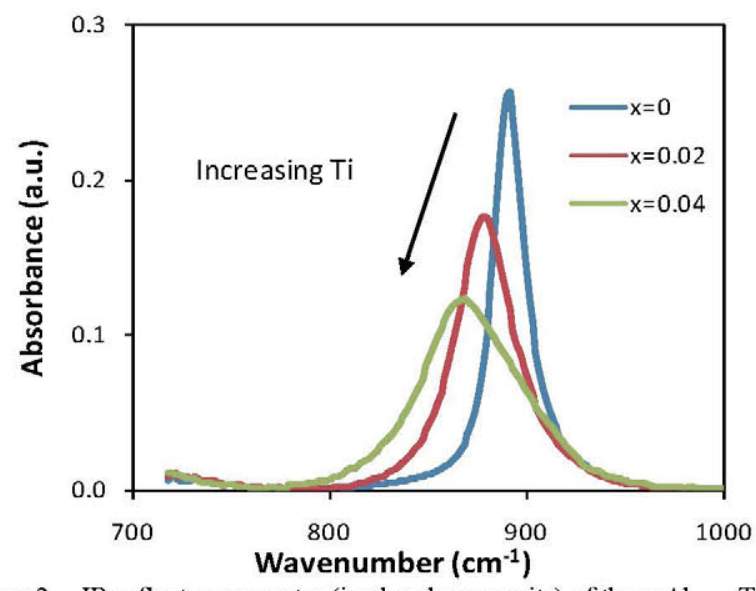

Figure 2. IR reflectance spectra (in absorbance units) of three $\mathrm{Al}_{(0.5-\mathrm{x})} \mathrm{Ti}_{\mathrm{x}} \mathrm{N}_{0.5}$ with $\mathrm{x}=0$ (blue), $\mathrm{x}=0.02$ (red) and $\mathrm{x}=0.04$ (green).

X-ray diffraction (XRD) patterns of the $\mathrm{Al}_{(0.5-\mathrm{x})} \mathrm{Ti}_{\mathrm{X}} \mathrm{N}_{0.5}$ films were measured in conventional Bragg-Brentano geometry in a high intensity Supratech XPert MRD diffractometer between $2 \theta=10^{\circ}$ and $2 \theta=80^{\circ}$ using the $\mathrm{K} \alpha_{1,2}$ doublet of a $\beta$-filtered $\mathrm{Cu}$ anode radiation. Because all the samples were strongly c-axis oriented, the rocking curves $(\mathrm{RC})$ around the $(00 \cdot 2)$ peak were also measured to assess the quality of the films through the value of its width at half maximum (FWHM). The XRD pattern of a representative $\mathrm{Al}_{(0.5-\mathrm{x})} \mathrm{Ti}_{\mathrm{x}} \mathrm{N}_{0.5}(\mathrm{x}=0.02)$ is depicted in figure 3 .

For assessing the electroacoustic performance of the material, bulk acoustic wave (BAW) test devices were fabricated by depositing Mo on top of $\mathrm{Al}$ :Ti:N/Mo on $\mathrm{Al} / \mathrm{Mo}$ acoustic reflectors with thicknesses of $\lambda / 4$ of the targeted resonant frequency of the devices. The piezoelectric activity of $\mathrm{Al}_{(0.5-\mathrm{x})} \mathrm{Ti}_{\mathrm{x}} \mathrm{N}_{0.5}$ films was assessed by measuring the electrical 
reflection coefficient $\left(S_{11}\right)$ at frequencies ranging from $10 \mathrm{MHz}$ to $10 \mathrm{GHz}$ using an Agilent PNA N5230A network analyzer.

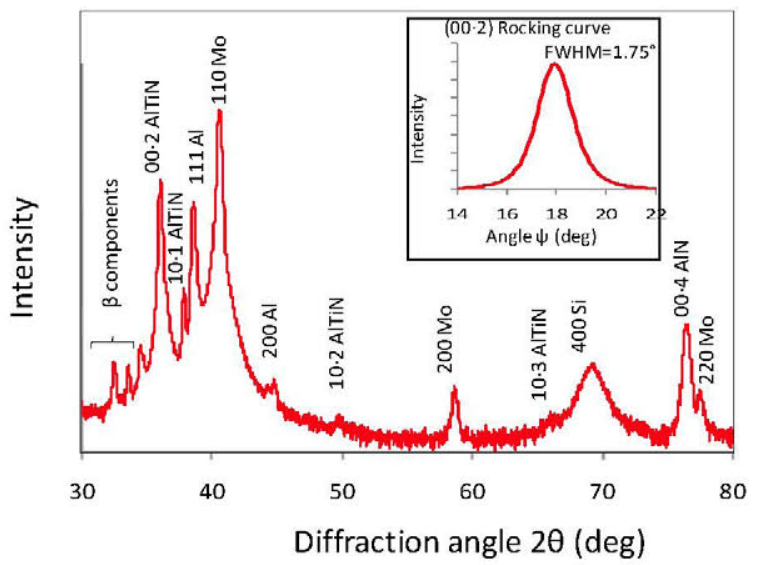

Figure 3. XRD pattern of a representative $\mathrm{Al}_{(0.5-\mathrm{x})} \mathrm{Ti}_{\mathrm{x}} \mathrm{N}_{0.5}(\mathrm{x}=0.02)$. The unlabeled peaks correspond to $\mathrm{Cu}-\mathrm{k} \beta$ radiation reflections. The inset displays the $\mathrm{RC}$ around the 00.2 direction.

The electrical impedance of the resonators, derived from $\mathrm{S}_{11}$, was fitted with Mason's model [14] to obtain accurate values of the material electromechanical coupling factor $\left(k^{2}\right)$. The simple reflector used had an acoustic transmittance of -17 $\mathrm{dB}$ at the central frequency, which yielded resonators with a quality factor $(Q)$ of around 20 . Despite the low value of $Q$, an accurate fitting of the impedance with frequency was achieved. Since the thickness of films, area of the resonator, dielectric constant $(\varepsilon)$ and sound velocity $\left(v_{s}\right)$ influence significantly the resonant frequency and the out-of-band response of the resonators, a careful assessment of all the geometrical dimensions of the devices was required to achieve reliable values of $\varepsilon$ and for each $\mathrm{Al}_{(0.5-\mathrm{x})} \mathrm{Ti}_{\mathrm{x}} \mathrm{N}_{0.5}$ film. The thicknesses of the electrodes and the piezoelectric film were carefully measured with a Veeco DekTak 150 profilometer in each device (after the electrical characterization) by patterning the $\mathrm{Al}_{(0.5-\mathrm{x})} \mathrm{Ti}_{\mathrm{x}} \mathrm{N}_{0.5}$ films by wet etching in warm $\mathrm{KOH}$ using the top electrode as etching mask. The area of each device was also carefully measured with an optical microscope with accuracy better than $1 \%$ to avoid possible undercutting effects in the top electrode patterning. The method allows assessing the electroacoustic properties of the $\mathrm{Al}_{(0.5-\mathrm{x})} \mathrm{Ti}_{\mathrm{x}} \mathrm{N}_{0.5}$ films independently of the design of the test device.

\section{RESULTS AND DISCUSSION}

\section{A. Composition}

The $\mathrm{Ti}$ content (x) in each $\mathrm{Al}_{(0.5-\mathrm{x})} \mathrm{Ti}_{\mathrm{x}} \mathrm{N}_{0.5}$ film varies monotonously $(\Delta x \sim 0.01)$ from the centre to the edge of the $150 \mathrm{~mm}$ wafer following a quasi-linear variation (see fig. 4).

\section{B. Structure}

Figure 5 shows the XRD patterns around the 00.2 peak for three $\mathrm{Al}_{(0.5-\mathrm{x})} \mathrm{Ti}_{\mathrm{x}} \mathrm{N}_{0.5}$ films of different composition. All the films studied have Ti contents lower than $6 \%$ and exhibit a wurtzite structure with a strong c-axis orientation. The $(00 \cdot 2)$ XRD peak shows an anomalous behavior when the Ti content exceeds $4 \%$. Above this value, the peak decreases in intensity and shifts to lower angles. Besides, the peak is actually composed of two components, one of which appears at lower angles and exhibits an intensity that increases with the $\mathrm{Ti}$ content.

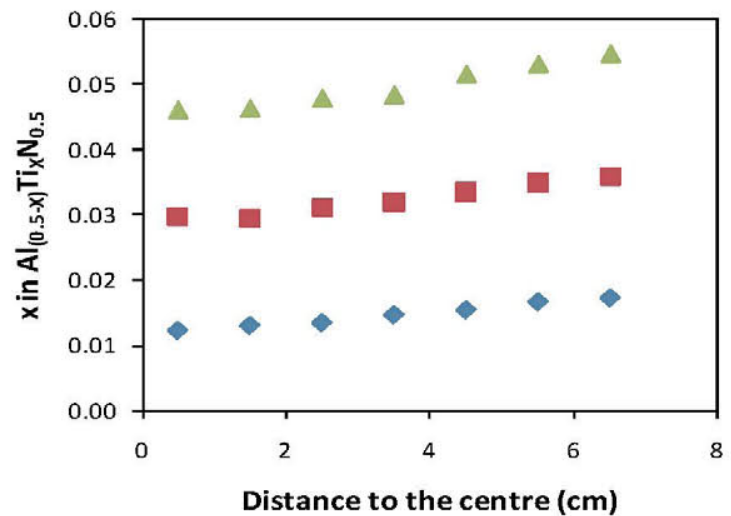

Figure 4. Radial distribution of Ti content in three samples at low, medium, and high power applied to the external Ti target.

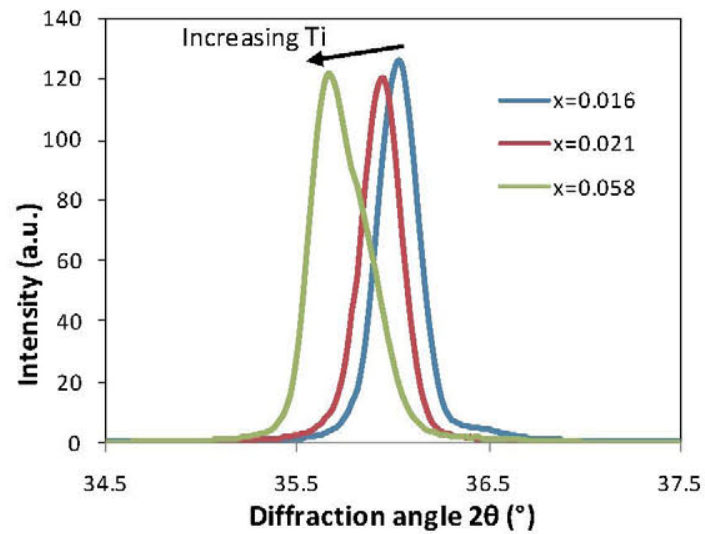

Figure 5. XRD patterns of three films with different composition: $\mathrm{x}=0.016$ (black), $x=0.021$ (red), and $x=0.058$ (green).

The evolution of the $(00 \cdot 2)$-peak diffraction angle and the intensity of its two components as a function of the Ti content are depicted in figure 6.

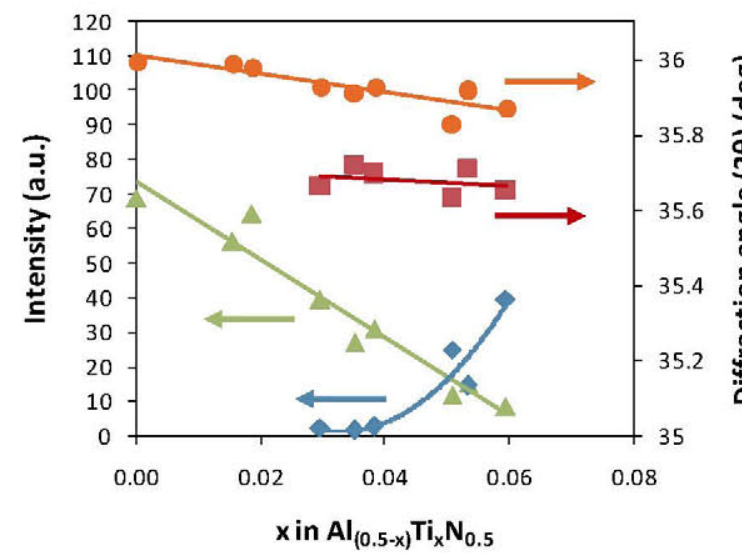

Figure 6. Diffraction angle and intensity of the two components of the $00 \cdot 2$ diffraction peak of $\mathrm{Al}_{(0.5-\mathrm{x})} \mathrm{Ti}_{\mathrm{x}} \mathrm{N}_{0.5}$ films with different $\mathrm{Ti}$ content. 
The existence of two diffraction contributions at close angles suggests the existence of two units cells with different $c$ spacing whose structural arrangement cannot be clearly deduced from the XRD patterns. Two possible arrangements could be: 1) two kinds of grains homogeneously distributed and 2) two juxtaposed layers of different composition. The splitting of the Ti-N absorption band observed in the IR reflectance spectra of Fig. 2 could also be explained with these two hypotheses. An additional characterization technique, such as transmission electron microscopy, would be necessary to assess the actual structure.

The FWHM of the rocking curve around the $00 \cdot 2$ peak varies from $1.75^{\circ}$ to $2.5^{\circ}$ without any relationship with the $\mathrm{Ti}$ content or the position in the wafer.

\section{Electroacoustic response}

The electrical response of the test resonators described in the experimental section were unambiguously fitted with the Mason's model [14], taking as variable parameters the electromechanical coupling factor $\left(k^{2}\right)$, the dielectric constant $(\varepsilon)$, and the longitudinal propagation velocity $\left(v_{1}\right)$ of the $\mathrm{Al}_{(0.5-\mathrm{x})} \mathrm{Ti}_{\mathrm{x}} \mathrm{N}_{0.5}$. The mass density of the deposited layers, deduced from the RBS measurements, is similar to that of pure AIN $\left(3300 \mathrm{~kg} \cdot \mathrm{m}^{-3}\right)$ within the uncertainty of the method. For the rest of the materials $\left(\mathrm{Mo}, \mathrm{Al}, \mathrm{SiO}_{2}\right)$ we have used the physical parameters deduced in previous works through the assessment of BAW devices based on pure AIN.

The incorporation of Ti atoms to the AlN lattice has a strong influence $k^{2}, \varepsilon$ and $v_{1}$. The variation of these parameters is shown in Fig. 7 as a function of the Ti content.

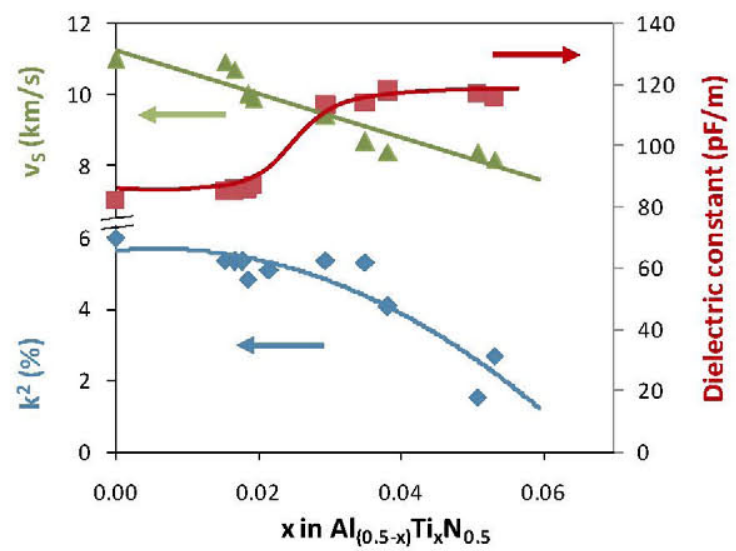

Figure 7. Electromechanical coupling factor $(\bullet)$, longitudinal velocity $(\boldsymbol{\Delta})$ and dielectric constant $(\mathbf{\square})$ of $\mathrm{Al}_{(0.5-\mathrm{x})} \mathrm{Ti}_{\mathrm{x}} \mathrm{N}_{0.5}$ as a function of $\mathrm{x}$.

The temperature coefficient of the resonant frequency (TCF) is slightly lower than the AlN one, although the high negative TCF of the metals forming the acoustic reflector impedes to give reliable values for the Ti-doped AlN films.

\section{CONCLUSIONS}

Ti-doped AlN films with Ti contents below 6\% have been deposited by co-sputtering two $\mathrm{Al}$ and $\mathrm{Ti}$ concentric targets. Different material properties, such as the crystal structure, piezoelectric activity, dielectric constant, and longitudinal velocity have been assessed as a function of the Ti content. The structural characterization of the films suggests that their composition is homogeneous along the thickness for $\mathrm{Ti}$ contents below $4 \%$ atomic and that the $c$-lattice parameter of the wurtzite structure increases as the Ti contents rises. Films with $\mathrm{Ti}$ contents greater than $4 \%$ atomic are very likely composed of two populations of grains with different $c$-lattice parameters values. The increasing of the Ti content in the films is accompanied by a rise of the dielectric constant, and a decrease of $k^{2}$ and $v_{1}$. However, a slightly improvement of the temperature coefficient of resonant frequency is found.

\section{ACKNOWLEDGMENT}

Work partially supported by the Spanish CICYT under the MAT2010-18933 project and by the Comunidad Autónoma de Madrid through its IV-PRICIT Programme.

\section{REFERENCES}

[1] Ferenc Tasnádi, Igor A. Abrikosov, and Ilia Katardjiev, "Significant configurational dependence of the electromechanical coupling constant of $\mathrm{B}_{0.125} \mathrm{Al}_{0.875} \mathrm{~N}^{\prime \prime}$, Appl. Phys. Lett., vol 94, 151911 (1-3), 2009.

[2] L. Liljeholm, M. Junaid, T. Kubart, J. Birch, L. Hultman, I. Katardjiev, "Synthesis and characterization of (0001)-textured wurtzite $\mathrm{Al}_{1-\mathrm{x}} \mathrm{B}_{\mathrm{x}} \mathrm{N}$ thin films", Surf. Coat. Technol., vol. 206, pp. 1033-1036, 2011.

[3] M. Akiyama, T. Kamohara, K. Kano, A. Teshigahara, Y. Takeuchi, and N. Kawahara, "Enhancement of piezoelectric response in scandium aluminum nitride alloy thin films prepared by dual reactive cosputtering", Adv. Mater., vol. 21, pp. 593-596, 2009.

[4] M. Moreira, J. Bjurstrom, I. Katarjev, and V. Yantchev, "Aluminum scandium nitride thin-film bulk acoustic resonators for wide band applications," Vacuum, vol. 86, pp. 23-26, 2011

[5] Q.Y.Wu, Z. G. Huang, R. Wu, and L. J. Chen, "Cu-doped AlN: a dilute magnetic semiconductor free of magnetic cations from first-principles study", J. Phys.: Condens. Matter., vol.19, pp. 056209 (1-6), 2007.

[6] Yasushi Endo, Takanobu Sato, Ayumu Takita, Yoshio Kawamura, and Masahiko Yamamoto, "Magnetic, electrical properties, and structure of $\mathrm{Cr}-\mathrm{AlN}$ and $\mathrm{Mn}-\mathrm{AlN}$ thin films grown on Ssi substrates", IEEE Trans. Magnet., vol. 41, pp. 2718-2720, 2005.

[7] D. Kumar, J. Antifakos, M.G. Blamire, Z.H. Barber, "High Curie temperatures in ferromagnetic Cr-doped AlN thin films", Appl. Phys. Lett. Vol. 84, pp. 5004-5006, 2004.

[8] V. V. Felmetsger and M. K. Mikhov, "Reactive magnetron sputtering of piezoelectric Cr-doped AIN thin films", 2011 IEEE International Ultrasonics Symposium Proceedings, pp. 835-839, 2011.

[9] J. T. Luo, B. Fan, F. Zeng and F. Pan, "Influence of Cr-doping on microstructure and piezoelectric response of AlN films", J. Phys. D: Appl. Phys., vol. 42, pp. 235406 (1-6), 2009.

[10] Ferenc Tasnádi, Igor A. Abrikosov, Lina Rogström, Jonathan Almer, Mats P. Johansson, and Magnus Odén, "Significant elastic anisotropy in $\mathrm{Ti}_{1-\mathrm{x}} \mathrm{Al}_{\mathrm{x}} \mathrm{N}$ alloys", Appl. Phys. Lett., vol. 97, pp. 231902, (1-3), 2010.

[11] David Holec, Richard Rachbauer, Li Chen, Lan Wang, Doris Lue, Paul $\mathrm{H}$. Mayrhofer, "Phase stability and alloy-related trends in $\mathrm{Ti}-\mathrm{Al}-\mathrm{N}, \mathrm{Zr}-$ $\mathrm{Al}-\mathrm{N}$ and $\mathrm{Hf}-\mathrm{Al}-\mathrm{N}$ systems from first principles", Surf. Coat. Technol., vol. 206, pp. 1698-1704, 2011.

[12] V. V. Felmetsger, P. N. Laptev, and S. M. Tanner, "Design, operation mode, and stress control capability of S-gun magnetron for ac reactive sputtering," Surf. Coat. Technol., vol. 204, pp. 840-844, 2009.

[13] V.V. Felmetsger and M.K. Mikhov " Reactive Sputtering of Highly CAxis Textured Ti-Doped AlN Thin Films", 2012 IEEE International Ultrasonics Symposium Proceedings, (to be published)

[14] J.F. Rosenbaum, "Bulk Acoustic Wave Theory and Devices", Artech House, Boston, London, 1988. 